Dear author,

Please note that changes made in the online proofing system will be added to the article before publication but are not reflected in this PDF.

We also ask that this file not be used for submitting corrections. 


\author{
D'Alessio Ilenia, ${ }^{1}$ Domanin Maurizio, ${ }^{2,3}$ Bellobuono Andrea, ${ }^{4}$ Boni Luigi, ${ }^{5}$ Cassinotti Elisa, \\ Romagnoli Silvia, ${ }^{3}$ Rolli Antonio, ${ }^{3}$ Del Gobbo Alessandro, ${ }^{6}$ and Trimarchi Santi, ${ }^{2,3}$ Milan, \\ Italy
}

\title{
Caveat for Vascular Surgeons: Lesson Learned from Acute Onset of a Rare Aortic Paraganglioma in a Young Boy
}

\begin{abstract}
Background: Paragangliomas (PGs) are rare neuroendocrine tumors arising from the extraadrenal autonomic paraganglia that are tiny organs formed by bundles of neuroendocrine cells derived from the embryonic neural crest and capable of catecholamines secretion. Diagnosis and treatment of aortic PGs could be a challenging issue when they present as an emergency setup (sudden abdominal pain and radiological images resembling a vascular emergency).

Case report: We present a rare case of a 16-year-old man with a symptomatic and bleeding left para-aortic mass, treated in emergency with embolization, before a staged videolaparoscopic resection. Histology of the mass showed the presence of a large aortic PG.

Conclusions: In case of active bleeding, in emergency, vascular consultants are always involved. Sometimes, circumstances are very atypical; therefore, it is essential to keep in mind rare pathologies. In such settings, multidisciplinary approach is primary to obtain a prompt diagnosis and appropriate treatment.
\end{abstract}

\begin{abstract}
Paragangliomas (PGs) are highly vascularized tumors, usually located along the sympathetic paravertebral ganglia of the thorax, abdomen, and pelvis and associated with blood vessels and neural structures. ${ }^{1}$ Sympathetic PGs often present with signs of catecholamine hypersecretion and less frequently as tumor masses. ${ }^{2}$ Frequently, PGs appear to be sporadic although one-third to one-half have inherited PG syndromes associated with germline mutations of the known susceptibility genes. ${ }^{3,4}$
\end{abstract}

Fondazione IRCCS $\mathrm{Ca}^{\prime}$ Granda Ospedale Maggiore PoliclinicoIstituto di Ricovero e Cura a Carattere Scientifico di natura pubblica via Francesco Sforza, 28-20122 Milano.

Disclosure The authors have no personal, financial, or institutional interest in any of the drugs, materials, or devices described in this article.

Q2 ${ }^{1}$ School of Vascular Surgery, University of Milan, Milan, Italy.

${ }^{2}$ Department of clinical and community science, University of Milan, Milan, Italy.

${ }^{3}$ Operative Unit of Vascular Surgery, Fondazione IRCCS Ca' Q3 Granda Ospedale Maggiore Policlinico.

${ }^{4}$ Operative Unit of Radiology Fondazione IRCCS $\mathrm{Ca}^{\prime}$ Granda Ospedale Maggiore Policlinico.

\section{CASE REPORT}

A 16-year-old boy, with no previous medical history or evidence of familiar genetic connective tissues diseases, was admitted in the emergency room (ER) in May 2018 with a 5-day history of abdominal pain, fever, and hematuria. In the last few hours before hospital admission, pain severely worsened reaching 9 in the pain visual analog scale. Body temperature was $37.5^{\circ} \mathrm{C}$, heart rate 98 beats/ $\mathbf{Q 4}$ min, and blood pressure 135/80 mm Hg. Physical examination of the abdomen was normal, with no masses or

${ }^{5}$ Operative Unit of General Surgery Fondazione IRCCS Ca' Granda Ospedale Maggiore Policlinico.

${ }^{6}$ Operative Unit of Pathology Fondazione IRCCS $\mathrm{Ca}^{\prime}$ Granda Ospedale Maggiore Policlinico.

Correspondence to: D'Alessio Ilenia, School of Vascular Surgery, University of Milan, Via Commenda 19, 20122 Milan, Italy; E-mails: ilenia.dalessio@gmail.com or ilenia.dalessio@unimi.it

Ann Vasc Surg 2020; $\mathbf{\square}: 1 . e 1-1 . e 7$

https://doi.org/10.1016/j.avsg.2019.12.016

(c) 2020 Elsevier Inc. All rights reserved.

Manuscript received: November 14, 2019; manuscript accepted: December 17, 2019; published online: 
111

112

113

114

115

116

117

118

119

120

121

122

123

124

125

126

127

128

129

130

131

132

133

134

135

136

137

138

139

140

141

142

143

144

145

146

147

148

149

150

151

152

153

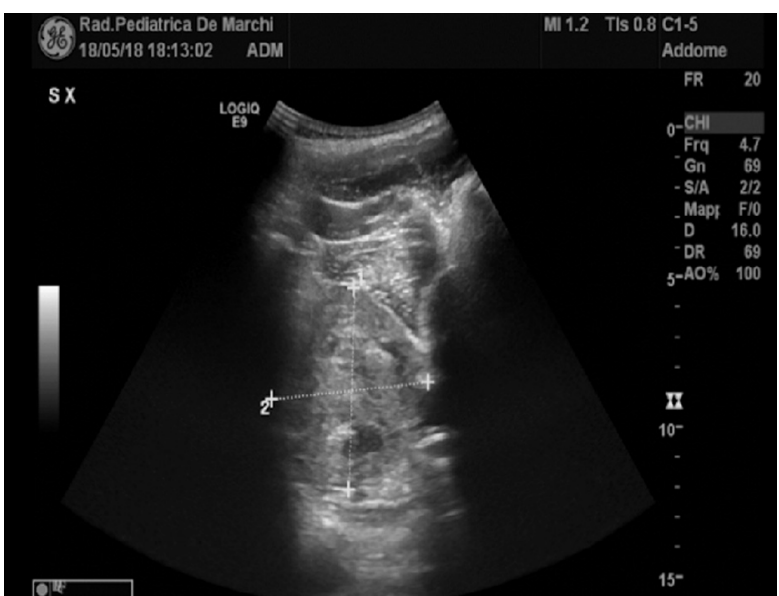

Fig. 1. Ultrasound scan image shows a roundish mass of $8 \times 7 \mathrm{~cm}$ near the aorta.

hepatosplenomegaly. Laboratory revealed mild leukocytosis with no others inflammatory markers. Initial management included intravenous fluid rehydration and painkiller drugs. A first abdominal ultrasound scan (US) examination revealed the presence of a left para-aortic ovoidal mass with $8.0 \times 7.0 \times 3.0 \mathrm{~cm}$ of diameter (Fig. 1). The patient was immediately submitted to computed tomographic angiography (CTA) that confirmed the presence of the mass. CTA also showed an active bleeding within the anatomical structure and inhomogeneous uptake of contrast medium with a direct communication with the aorta (Fig. 2). ER doctors immediately asked for vascular surgery consultation.

With a multidisciplinary approach (anesthetists, interventional radiologists, general, vascular, and pediatric surgeons), several hypotheses of differential diagnosis were discussed. The patient was an under-18 volleyball player, and his parents reported a violent crash occurred during a match against the telescopic post some weeks ago, but no further investigation about it was performed. The hypothesis of a post-traumatic aortic pseudoaneurysm was then suggested. Another hypothesis included the oncological nature of the mass, and thereafter, in the suspicion of the para-aortic PG, plasma normetanephrine, oncogene markers, chromogranin A, urinary noradrenaline, and dopamine were tested, resulting negative. Based on the persistence of severe abdominal pain with the high risk of hemorrhagic shock owing to continuous bleeding, the patient was submitted to urgent embolization of the mass.

Through a percutaneous bilateral femoral arterial access, a selective angiography of the aorta was carried out, using Isovue-250 (Iopamidol, Bracco, Milan, Italy)

Q5 and a 65-cm 5F Performa sizing Pigtail catheter (Merit Medical, South Jordan). Angiography showed an active bleeding from a vessel surrounding the mass, directly originating from the aorta, near the left spermatic artery. The right femoral artery was engaged with a 4F Cobra catheter (Terumo, Tokyo, Japan) and then with a $4 \mathrm{~F}$ Radifocus renal catheter (Terumo, Tokyo, Japan) to select the left renal artery. The vessel that surrounded the mass was embolized with 7 microcoils 2-25 0,018" Extrusion coils (Bard, Covington) using a 2.7 F Progreat ${ }^{\circledR}$ microcatheter system (Terumo, Tokyo, Japan). Finally, the vessel's origin was closed using Spongostan (Somerville, New Jersey), sparing the spermatic artery (Figs. 3-4). No signs of bleeding were evident at the end of procedure. The patient was clinically stable during the operation, and the total length of procedure was $1 \mathrm{hr} 30 \mathrm{~min}$ with a time of Xray exposure of $40 \mathrm{~min}$.

After this first step, hemoglobin values and others vital parameters remained stable; nevertheless, the patient continued complaining abdominal pain. A CT scan, performed after embolization procedure, revealed a compression and dislocation of the left ureter, with signs of hydronephrosis and a dilatation of the left testicular vein. Suspecting the presence of both an aortic PG and a malignancy, the multidisciplinary team decided to treat the patient by means of a videolaparoscopic (VLS) approach. The preoperative alpha-adrenergic blockade was necessary before the surgical manipulation of the mass to avoid hypertensive crisis. VLS was performed with the patient placed in the right flank position. Three trocars $(10 \mathrm{~mm})$ in the abdominal midline and one trocar in the left hypochondrium were located. At initial examination of the abdominal cavity, voluminous left paraaortic mass arising in the contest of the left mesocolon was found, dislocating posteriorly main renal vessels. The parietal peritoneum was divided, and the paraaortic lesion was dissected from the aortic plane, medial to lateral, and from below to the top, preserving the inferior mesenteric vessels. The mobilization was performed up to the splenic vein. Several small vessels, supplying the mass and arising directly from the aorta, were isolated and ligated with vascular clips. The mobilization was completed through difficult dissection from aortic plane and mesocolic posterior surface. The perfusion of the colon was verified with fluorescence angiography. Specimen, a large brownish-red mass, was extracted through a Pfannenstiel minilaparotomy in an endobag. A drain was left in the pelvis (Fig. 5A).

No intraoperative complications occurred, and postoperative course was uneventful. The specimen of the lesion was described as an oval mass of $7.8 \times$ $7.0 \times 2.8 \mathrm{~cm}$, with a grayish-white color, soft, diffusely hemorrhagic on the cut surface (Fig. 5B). Microscopically, the lesion resulted as a capsulated proliferation of medium-sized cellular elements, with moderate nuclear pleomorphism and relatively large, clear or eosinophilic cytoplasm, organized in solid nests delimited by thin fibrous septa in which ecstasic microvascular Q6 structures were present. Necrosis, likely iatrogenic (probably caused by preoperative tumor embolization), was present inside. Proliferative index measured by Ki67 antibody was 5\%. There was no vascular invasion. The neoplastic elements resulted immunoreactive for the antibodies anti-vimentin chromogranin $\mathrm{A}$ and synaptophysin and negative for HMB45, CD31, CD34, 


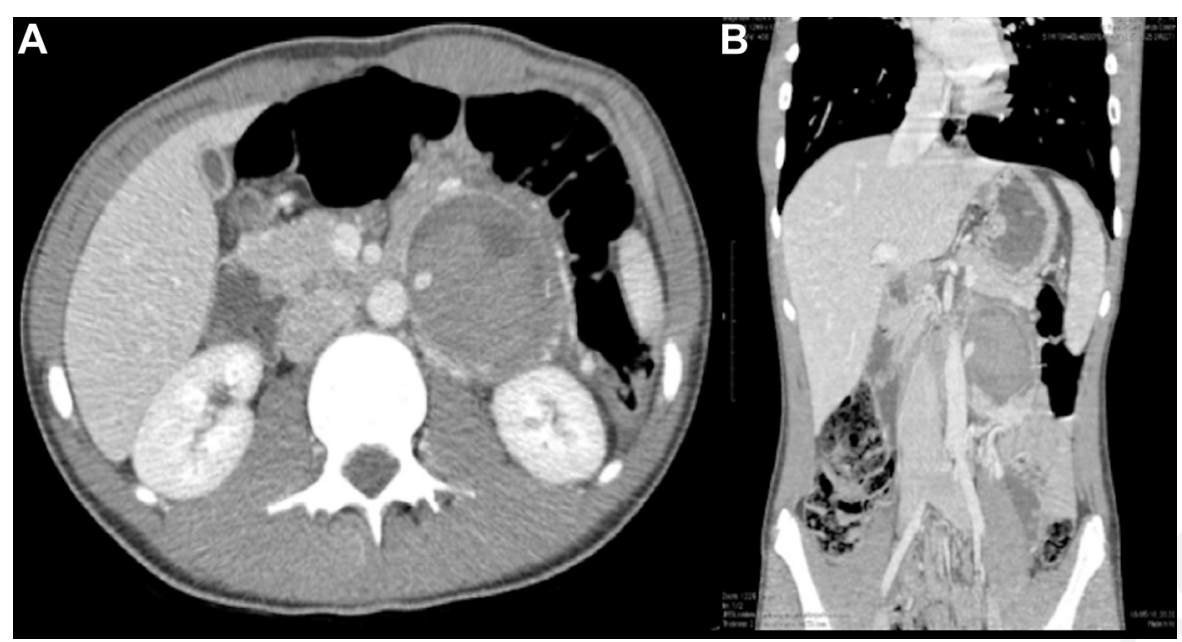

Fig. 2. (A) The image shows an axial section of the abdomen with the presence of large mass, near the aorta. (B) The image shows a sagittal reconstruction of the computed tomography; active bleeding inside the mass is observed.

and NF1. In 2011, loss-of-function mutations in

pan-cytokeratin, and S100, confirming the diagnosis of PG. The genetic study was negative for main mutations. (Fig. 6). During the 16-month follow-up period, no signs of recurrence or metastasis were detected. Radiographic imaging (CTA) of the primary site at discharge, 3 months postresection, and 1 year later were performed. Every 6 months, biochemical test for catecholamine, accurate history and physical examination, and monitoring of blood pressure and routine biochemical markers were made. The pathologists classified the mass as a sporadic one; therefore, family did not need further genetic tests.

\section{DISCUSSION}

PGs are rare neoplasms, with an approximately estimated annual incidence of 0.8 per 100,000 persons per year. ${ }^{5}$ Most of them are diagnosed within the third to the fifth decades. The male to female ratio is approximately equal among patients with hereditary PG, whereas sporadic tumors are much more common in women $(71 \%$ vs. $29 \%) .{ }^{6}$ Most hereditary PGs, particularly those arising in the skull base and neck, have been linked to mutations in the genes encoding different subunits of the succinate dehydrogenase (SDH) enzyme complex. In addition, susceptibility to pheochromocytomas and PGs is an established component of four genetic syndromes: multiple endocrine neoplasia types $2 \mathrm{~A}$ and $2 \mathrm{~B}$, neurofibromatosis type $\mathrm{l}$ (NF1), von Hippel-Lindau (VHL), and Carney-Stratakis dyad. Most cases of hereditary PG are accounted for mutations in $S D H D, S D H B$, and $S D H C, V H L$, the MAX (MYC-associated factor X) gene were identified in patients with familial pheochromocytoma. The MAX gene is located on chromosome 14q23.3. It encodes MAX protein, which belongs to the basic helix-loop-helix leucine zipper (bHLHZ) family and which interacts with MYC and MXDl to form the MYC-MAX-MXDl network of transcription factors that regulate cell proliferation, differentiation, and apoptosis. ${ }^{7}$ Most of PGs are benign, whereas the malignant ones are defined by their metastatic behavior because it is difficult to predict histologically the malignancy. Approximately $75 \%$ of sympathetic PGs are located Q7 in the abdomen, at the junction between the vena cava and left renal vein, or at the organ of Zuckerkandl, located at the aortic bifurcation, close to the inferior mesenteric artery.

As shown by this case, diagnosis can be very challenging, in particular when requested in an emergent condition. Moreover, clinical presentation is variable and depends on tumor location and catecholamine secretion. Most sympathetic PGs are functional and present with catecholamine hypersecretion, whereas a minority, as in this case, present with pain or other symptoms related to mass effect.

As first-line imaging, abdominal US is the most commonly used test, whereas CTA, magnetic resonance imaging, or arteriography are usually performed to clarify or confirm initial diagnosis. Generally, abdominal PGs are characterized by high degree of vascularity, being located close to the sympathetic chain. These findings usually allow 


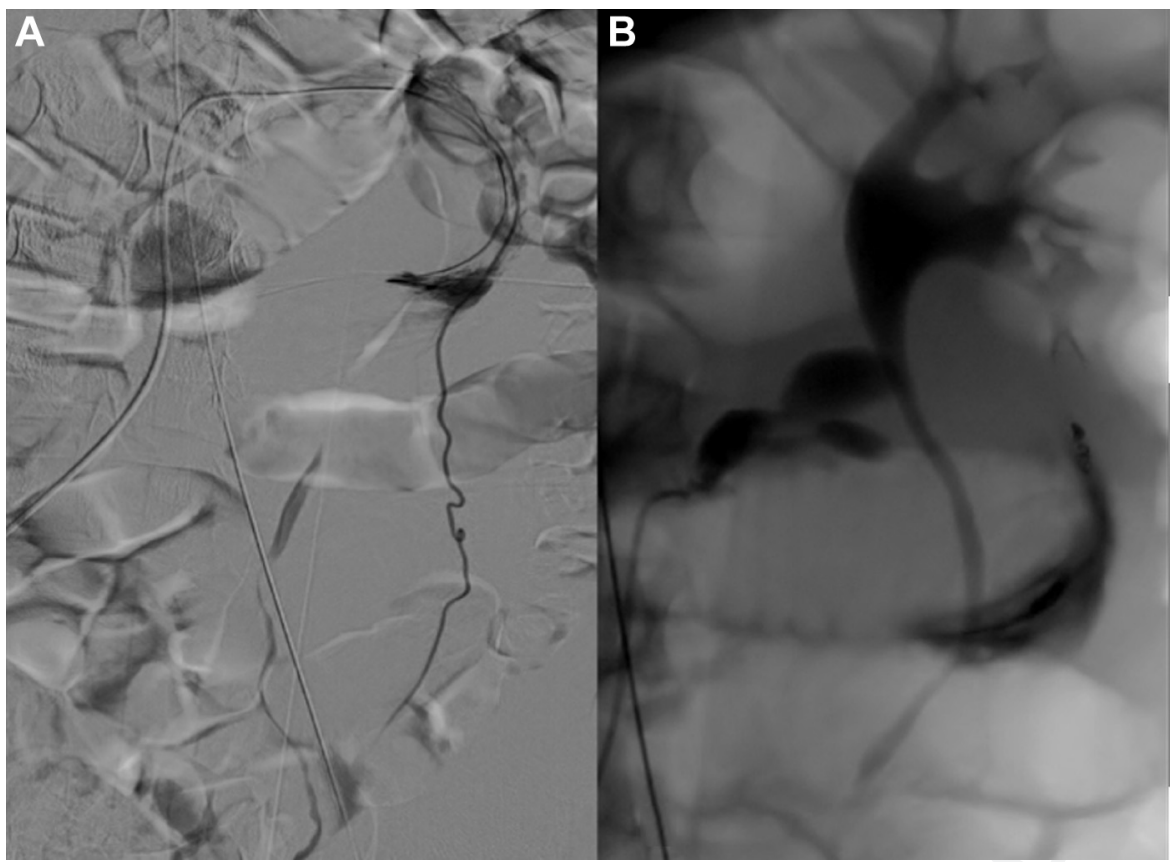

Fig. 3. (A) The image shows an active bleeding from the mass and the course of an artery originating from the spermatic artery. (B) The image shows the presence of

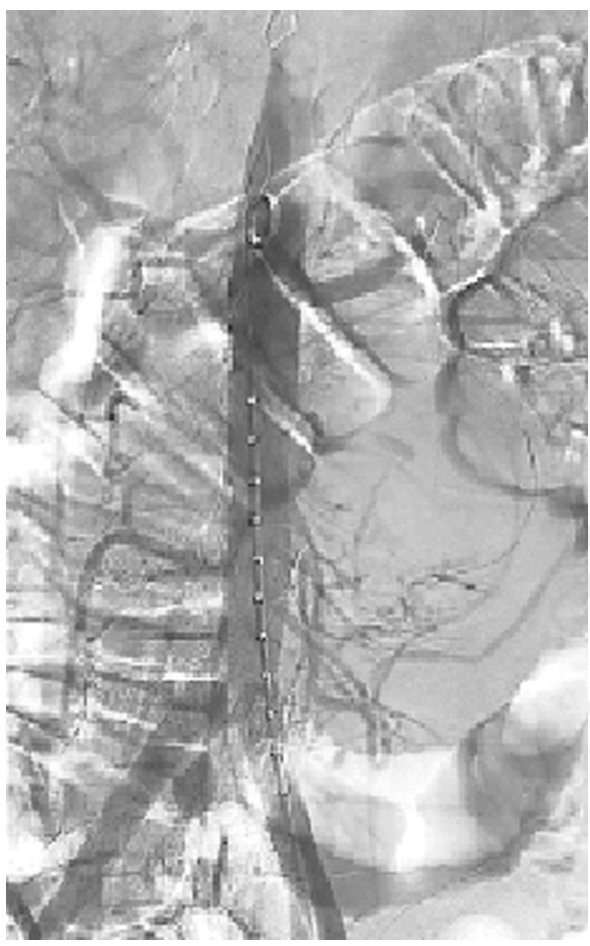

Fig. 4. The image shows the final angiogram, with no active bleeding.

performing preoperative diagnosis, even if PGs can be confused with different clinical conditions and misdiagnosed.
7 distal microcoils in the lumen of the artery and an active bleeding from its origin, near the aorta.

This case, owing to the number and nonspecific nature of the symptoms presented, has made the differential diagnosis a challenging process. After CTA, three different diagnostic hypothesis were made: (1) retroperitoneal sarcoma or other malignancy due to apparent infiltrative behavior of the mass with active bleeding from the aorta, (2) spontaneous post-traumatic or cryptogenic aortic pseudoaneurysm, and (3) aortic PG. Considering the age of the patient and the potential lifethreatening condition, a multidisciplinary group discussion was setup, along with other specialists involved, such as general surgeons, oncologists, and pediatricians. Operative roadmap was discussed and approved. Emergency aortography resulted helpful because it ruled out the hypothesis of aortic pseudoaneurysm and allowed to selectively embolize the mass, preparing it to successive VLS removal. By the way, it did not solve the diagnostic doubt between malignancy and PG. Incisional or needle aspiration (NA) biopsy was excluded in the fear of severe hypertension due to adrenergic crisis, in case of catecholaminesecreting PG. Furthermore, NA has a poor diagnostic value for PG and can lead to misdiagnosis with many different neoplasms, including fibroma, fibrosarcoma, or also malignant melanoma. ${ }^{8}$ In addition, NA may result in severe hemorrhage of the mass or seeding of neoplastic cells.
407

408

409

410 


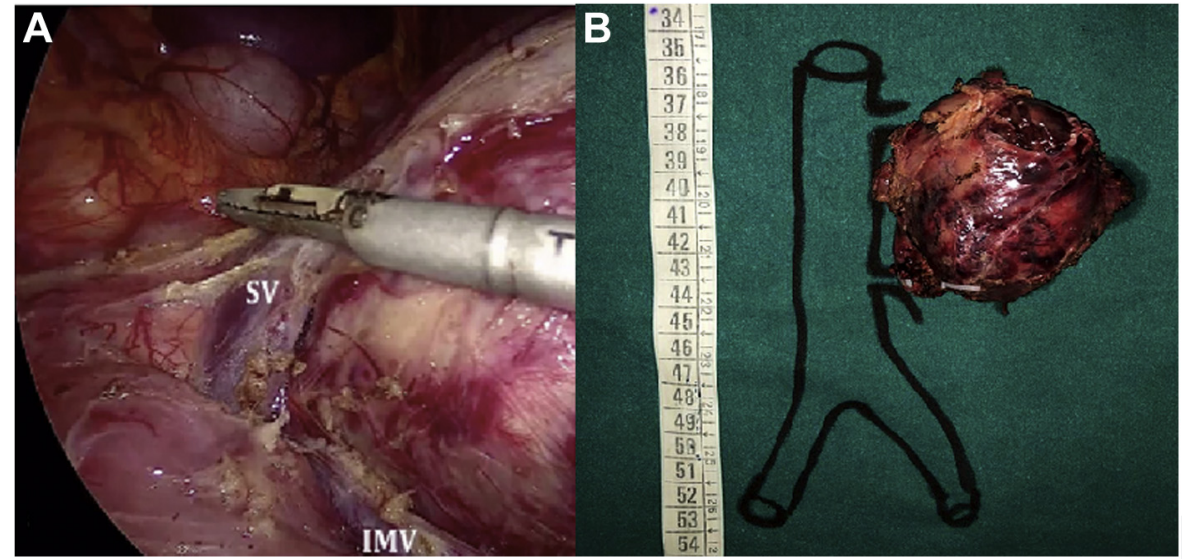

mass of $7.8 \times 7.0 \times 2.8 \mathrm{~cm}$, after VLS resection, with a

Fig. 5. (A) The images shows a frame of VLS resection, we can appreciate the spermatic vein (SV) and inferior mesenteric vein (IMV). (B) The image shows the oval grayish-white color, soft, and diffusely hemorrhagic on the cut surface. VLS, videolaparoscopic.
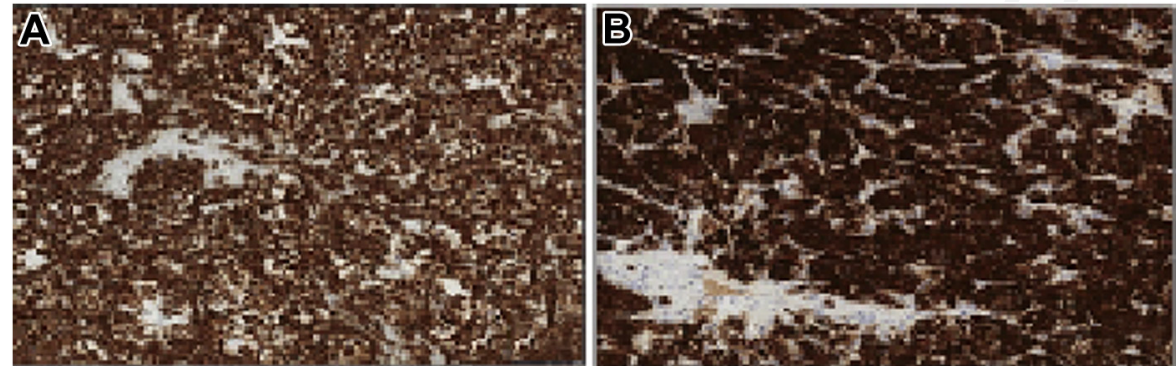

516

517

518

519

520

521

522

523

524

525

526

527

528

529

530

531

532

533

534

535

Fig. 6. Immunohistochemical features of the neoplastic with antibodies against HMB45 (C) and pan-cytokeratin (D) antigens (original magnifications: $50 \times$ ).

Considering the young patient's age and the uncertainty of diagnosis, we have chosen, in accordance with general surgeons, to proceed with VLS exploration. This approach is associated with a shorter hospital stay and a rapid return to normal activity.

In literature, few studies of para-aortic and/or paracaval VLS resection for retroperitoneal lesions have been reported. Misra et al. ${ }^{9}$ reported successful treatment in four cases of laparoscopic resection for retroperitoneal tumors. Walz et al. ${ }^{10}$ resected 27 retroperitoneal PGs in 19 patients by means of VLS, describing one local recurrence. Other authors have published individual case reports on the treatment of unusual retroperitoneal lesions, demonstrating the feasibility of VLS resection. ${ }^{1-13}$ Chung et al. ${ }^{14}$ observed that VLS is feasible only for selected patients.

Life-long follow-up is important in all patients after PG resection. A significant proportion of patients
541

542

543

544

545

546

547

548

549

550 


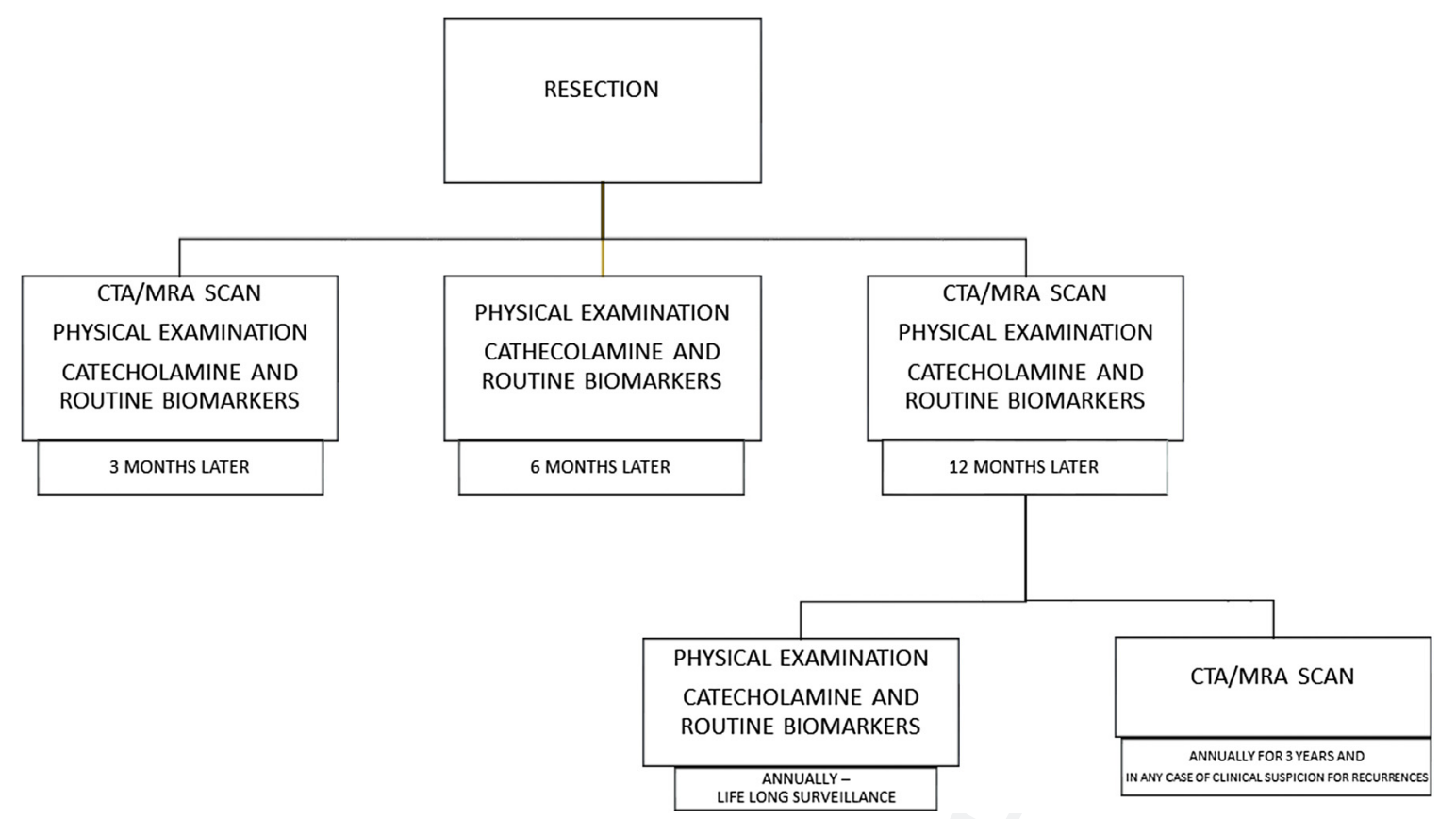

Fig. 7. Follow-up algorithm. CTA, computed tomography angiography; MRA, magnetic resonance angiography.

with apparently benign PG $(31 \%$ in one combined series of benign skull base and neck, thoracic, and abdominal PG) will have persistent or recurrent disease or develop metachronous primary tumor(s). ${ }^{15}$ We suggest radiographic imaging (CTA/magnetic resonance anigiography [MRA]) of the primary site and biochemical testing for catecholamine secretion, three months after resection, and accurate physical examination, monitoring blood pressure, and biochemical routine markers every 6 months for the first three years. Then, life-long annual biochemical testing might be needed, using imaging studies only if clinically indicated. The choice of imaging strategy is empiric. We performed CTA during the first year and MRA for the subsequent years to avoid irradiation. (Fig. 7).

The multidisciplinary collaboration resulted essential for the correct management of the case. $P G$ is a rather uncommon disease, and there is no consensus on who has to take in charge of the patient. Usually, in an elective setting, PGs are treated by general surgeons, but our case shows the unpredictable behavior of this pathology and the uncertainty of a prompt diagnosis in an emergency setting.

\section{CONCLUSION}

Para-aortic PGs and related complications, (i.e., bleeding in our case), are rare clinical findings, particularly in young patients without history of genetic disorder or previous symptomatology.

In elective settings, patients with para-aortic PGs are managed by general/pediatric surgeons via the laparoscopic approach. From the vascular surgeon's perspective, the key to successful elective PG resection is to provide support in case of intraoperative complications, such as a major bleeding from the aorta or inferior vena cava, requiring open conversion. In an emergency setting, particularly in case of unusual clinical presentation, diagnosis and decision making for the best treatment option become challenging. Our case taught us how rare pathologies and related potential life-threatening complications require specific skills that belong to various figures (vascular surgeons, general surgeons, interventional radiologists, pediatric surgeons, and anesthetists) and how teamwork is crucial to obtain successful clinical results. Moreover, this case report might be considered a caveat for all the vascular specialists, in case of the unusual observation of paraaortic masses in young patients. 
pheochromocytoma and paraganglioma. J Clin Endocrinol Metab 2010;95:2023-37.

3. Burnichon N, Brière J-J, Libé R, et al. SDHA is a tumor suppressor gene causing paraganglioma. Hum Mol Genet 2010;19:3011-20.

4. Fishbein L, Merrill S, Fraker DL, et al. Inherited mutations in pheochromocytoma and paraganglioma: why all patients should Be offered genetic testing. Ann Surg Oncol 2013;20:1444-50.

5. Beard CM, Sheps SG, Kurland LT, et al. Occurrence of pheochromocytoma in rochester, Minnesota, 1950 through 1979. Mayo Clin Proc 1983;58:802-4.

6. Boedeker CC, Neumann HPH, Maier W, et al. Malignant head and neck paragangliomas in $S D H B$ mutation carriers. Otolaryngol Head Neck Surg 2007;137:126-9.

7. Comino-Méndez I, Gracia-Aznárez FJ, Schiavi F, et al. Exome sequencing identifies MAX mutations as a cause of hereditary pheochromocytoma. Nat Genet 2011;43: 663-7.

8. Chuah KL, Tan PH, Chong YY. Test and teach. Number ninety-three: Part 1. Carotid body paraganglioma. Pathology 1999;31:215-6.
9. Misra MC, Bhattacharjee HK, Hemal AK, et al. Laparoscopic management of rare retroperitoneal tumors. Surg Laparosc Endosc Percutan Tech 2010;20:e117-22.

10. Walz MK, Alesina PF, Wenger FA, et al. Laparoscopic and retroperitoneoscopic treatment of pheochromocytomas and retroperitoneal paragangliomas: results of 161 tumors in 126 patients. World J Surg 2006;30:899-908.

11. Kelliher K, Santiago A, Estrada DE, et al. Laparoscopic excision of a familial paraganglioma. J Laparoendosc Adv Surg Tech A 2009;19(Suppl 1):S155-8.

12. Najah H, Ayed A, Noullet S, et al. Laparoscopic resection of interaortocaval paraganglioma in left lateral decubitus (with video). J Visc Surg 2017;154:459-60.

13. Abe T, Sazawa A, Harabayashi T, et al. Laparoscopic resection of paraaortic/paracaval neurogenic tumors: surgical outcomes and technical tips. Surg Endosc 2016;30:4640-5.

14. Chung L, O'Dwyer PJ. Laparoscopic resection of paraaortic or paracaval lesions: feasibility and outcome. Surg Endosc 2013;27:4153-6.

15. Erickson D, Kudva YC, Ebersold MJ, et al. Benign paragangliomas: clinical presentation and treatment outcomes in 236 patients. J Clin Endocrinol Metab 2001;86:5210-6. 